\title{
OBISPOS EFÍMEROS, COMUNIDADES Y HOMICIDIO EN LA RIOJA ALTA EN LOS SIGLOS X Y XI'
}

\author{
Juan José Larrea \\ Universidad del País Vasco. Vitoria-Gasteiz
}

\section{Del Pirineo a Tobía}

El pueblo de Tobía se encuentra aguas arriba del río Najerilla, recostado en las estribaciones de la Sierra de La Demanda. Desde el punto de vista de su presencia en las fuentes, su obispado es probablemente el más efímero de la España cristiana. Hay otras referencias únicas a obispos en las regiones cercanas de Álava o Cantabria, pero no a sedes. Y sin embargo, su aparición no tiene lugar en una oscura donación o en la críptica referencia a la consagración de una humilde iglesia. Figura en la noticia de algo que hubo de tener realce y eco, puesto que se trató de la consagración de tres obispos para el reino de Pamplona que acababa de ampliarse con la conquista de la Rioja Alta y la incorporación de Aragón. La paradoja no carece de interés.

El texto en cuestión es la segunda entrada de una pequeña nómina y obituario de obispos que rigieron la sede de Pamplona en tiempo de Sancho Garcés I y de sus sucesores, incluida en el Códice de Roda. Forma parte pues de los que J.M. Lacarra Ilamó "textos navarros" de este códice, es decir de materiales literarios e historiográficos guardados o producidos en el entorno de la corte real de Nájera a finales del siglo X. Como en la genealogías del mismo rotense, el autor ha preferido dejar en blanco las fechas que ignora -que son casi todasa inventarlas. La entrada que nos interesa es la del obispo Galindo. Es la más

1. Este artículo forma parte del Proyecto de Investigación "La apropiación del territorio: lógica comunitaria y conflicto entre los siglos VIII y X", financiado por el MEC (HUM 200762038/HIST). En buena medida complementa el análisis político y geoestratégico propuesto por D. Peterson en este mismo número. 
rica en informaciones, pero una rotura en el pergamino impide la lectura del comienzo de algunas líneas. He aquí el texto de ésta y de la anterior, según la transcripción de J.M. Lacarra²:

[ ] VIII. Mense decembrio, obiit famulus Dei Basilius episcopus et quiescit in Illerde.

[ ]X. successit post eum Galindus episcopus XL etatis sue anno et ordinatis [ ] II episcopis, id est, Sisuldus episcopus Calagorriensis, Teudericus episcopus Tubiensis [ ] us episcopus Sisabensis.

La $X$ que sigue visible a la derecha de la rotura debe corresponder a la Era de 960, pues el obispo Galindo figura en un documento de Siresa de 922 y Basilio gobierna aún la diócesis en $921^{3}$.

La explicación en vigor sigue siendo la que propuso A. Ubieto en 1954². La rapidísima expansión del reino en tiempos de Sancho Garcés reclamaba una reorganización paralela en el ámbito eclesiástico, cuyo gobierno seguía dependiendo de un solo obispado, el de Pamplona. La ordenación de tres obispos sería en realidad el acto fundador de tres diócesis: Aragón con sede en Sasabe, Calahorra y Tobía. La primera sería de nuevo cuño, pues hasta entonces, conjeturaba Ubieto, los valles del primitivo Aragón habrían dependido del obispado pamplonés. La segunda sería naturalmente la restauración de la vieja sede visigoda de Calahorra, de la que no se tienen noticias desde la mención del obispo Recaredo en 812 ¿Y la tercera? Ubieto se limita a decir que Tobía y Calahorra debieron fundirse pronto en la sede de Nájera-Calahorra. J.M. Lacarra, que en 1945 ni siquiera estaba seguro de la idenficación con Tobía ${ }^{5}$ y J. Goñi, admitieron después la tesis de Ubieto, con dos matices. En Tobía quizá se trataba de restaurar la diócesis de Oca -pensaba Lacarra-, y en cualquier caso, los dos obispados riojanos no prosperaron y acabaron fundiéndose en el de Nájera ${ }^{6}$. Más recientemente, A.J. Martín Duque mantiene la interpretación general relativa a la creación de tres nuevas sedes acordes con la ampliación del reino. Tobía se explica como la instauración de una nueva sede que responde al crecimiento urbano habido en Nájera bajo dominación musulmana y que se instala "con centro al principio en un lugar simbólico, Tobía, pero referido al cabo

2. LACARRA, J.M., "Textos navarros del Códice de Roda", Estudios de Edad Media de la Corona de Aragón 1 (1945) 193-283: p. 262-265.

3. GOÑI, G., Historia de los obispos de Pamplona. S. IV-XIII, Pamplona, 1979, p. 92-95.

4. UBIETO, A., "Las diócesis navarro-aragonesas durante los siglos IX y X", en: A. Ubieto, Trabajos de investigación, Valencia, 1972, p. 31-51.

5. LACARRA, "Roda" (cit. n. 2) p. 264.

6. LACARRA, J.M., Historia política del reino de Navarra desde sus orígenes hasta su incorporación a Castilla, Pamplona, 1973, t. 1, p. 170-171; GoÑı, Obispos (cit. n. 3) p. 95. Sigue a ambos E. SAínz RIPA, Sedes episcopales de la Rioja. Siglos IV-XIII, Logroño, 1994, p. 172-173. 
de muy poco tiempo a la nueva civitas organizadora en el plano socio-económico del territorium Nagerense ${ }^{\prime \prime}$. Qué relación pudiera tener Tobía con Oca, que ni siquiera estaba en territorio del rey de Pamplona, o por qué se crea una sede para Nájera y se establece en Tobía, o qué valor simbólico tiene esta localidad, es cosa que no se ha explicado. La incomodidad con respecto a la aparición de Tobía en esta triple ordenación no ha hecho sino crecer con el paso de los años. Sólo en la última obra de síntesis de F.J. Fernández Conde se sugiere una explicación más vinculada a la realidad previa de los territorios incorporados que a una creación ex novo decidida por los pamploneses ${ }^{8}$.

No es menor la incomodidad que produce un examen de la cronología. Puesto que un obispo Ferriolo efectúa una cesión de derechos en el valle de Echo a favor de S. Pedro de Siresa en 922, y lo hace en presencia del obispo Galindo de Pamplona', la creación de sedes habría tenido que hacerse en el mismo 922, entre el acceso de Galindo a la cátedra y el acto de Siresa. Ahora bien, la toma definitiva de Nájera por Ordoño II de León no se produce hasta 923 y lo más probable es que pasara a manos navarras sólo tras la muerte del leonés en $924^{10}$. En tales circunstancias, no es imposible que Galindo de Pamplona consagre un prelado para Calahorra en previsión de su conquista por los navarros, pero extraña mucho que consagre un obispo cuya sede es una aldea situada no ya bajo control musulmán, sino en la zona de conquista de los aliados leoneses.

Pensamos sin embargo que es justamente en la cronología donde asoma el hilo que quizá permita ir desenredando la madeja. El documento que limita la triple consagración a unos meses de 922 es la ya citada noticia de Aragón ${ }^{11}$. No es una noticia cualquiera: es la que se escogió para abrir el cartulario de Siresa. Se trata de un pequeñísimo cartulario en letra visigótica hoy desaparecido

7. MARTíN DUQUE, A.J., "El reino de Pamplona ", en: J.M. Jover (ed.), La España cristiana de los siglos VIII al XI. Los núcleos pirenaicos (718-1035). Navarra, Aragón, Cataluña (Historia de España Menéndez Pidal, 7/2) Madrid, 1999, p. 114 y 242.

8. FERNÁNDEZ CONDE, F.J., La religiosidad medieval en España. Alta Edad Media (siglos VII-X), Oviedo, 2008, p. 137. En lo que se refiere al obispado de Aragón, sin embargo, el autor enfoca más bien la problemática en la relación entre los obispados aragoneses altomedievales y la antigua sede de Huesca y acepta la idea de Ubieto que hace depender los valles del primitivo Aragón del obispado de Pamplona (op. cit., p. 141).

9. UBIETO, A., Cartulario de Siresa, Valencia, 1960, nº 6 .

10. GARCÍA DE CORTÁZAR, J.A., “¿Ocupación o dominación cristiana de la rioja en los años 920 a 925" , en: J.A. Sesma (coord.), Historia de la ciudad de Logroño, Logroño, 1994, p. 59-62: p. 62; MARTíN DUQUE, "Pamplona" (cit. n. 7) p. 115. Véase la contribución de D. Peterson en este número.

11. Conviene aquí recordar que la historia eclesiástica de Aragón en este período ha sido prácticamente abandonada por la investigación reciente: LALIENA, C., "Problemas historiográficos de la Alta Edad Media aragonesa: una revisión crítica", Argensola 113 (2003) 13-35. 
que sólo conocemos a través de copias del siglo XVIII ${ }^{12}$. El motivo de su elección es sencillo. Se trata del más antiguo acto documentado de un rey en favor del monasterio. De hecho, los tres primeros textos copiados en el cartulario fueron aquellos relacionados con los reyes de Pamplona, mientras que los producidos por los condes de Aragón, con ser más antiguos, quedaron relegados a un lugar posterior. El de 922 es una simple noticia, cosa excepcional en el cartulario porque el resto de documentos de reyes, condes o magnates son instrumentos de donación o de confirmación. Y no está compuesta con mucho cuidado por parte del redactor ${ }^{13}$, lo que indica que no ha habido voluntad de falsificar o de reconstruir un hipotético diploma perdido, pues para ello no faltaban materiales en el scriptorium pirenaico ${ }^{14}$. El contraste entre la importancia del acto de 922 y su descuido formal nos Ileva a pensar que no generó un diploma. Lo cual cuadra con lo que se hizo en aquella circunstancia. En efecto, el conde Galindo Aznar I había dotado en 867 a Siresa con derechos en todo el valle de Echo, desde Javierregay hasta los puertos ${ }^{15}$. Después, Galindo Aznar II, quizá de manera simultánea a la ceremonia de 922, volvió a dar al monasterio las tierras y ganados que ya había dado su predecesor ${ }^{16}$. Y lo que hace Sancho Garcés de Pamplona en 922 no es sino volver a confirmar lo que en principio ya era de Siresa desde un par de generaciones antes. Da la impresión de que lo fundamental era escenificar la nueva arquitectura del poder en Aragón, con una ceremonia en la que ofician por parejas el rey de Pamplona y el conde de Aragón, el obispo de Pamplona y el de Aragón:

... donatione quam dedit Sancio Garcianes ad domnum Petrum de Syresia, et domnus Galindo comes: et dederunt ad Syresiam de foze in suso, ad serviendum Deo et domno Petro. Et domnus Ferriolus episcopus

\section{UBIETO, CartSiresa.}

13. Da por ejemplo la sensación de que el escriba ha copiado parcialmente una fórmula de suscripción olvidándose de poner su nombre: Ego Rogitus scripsi hanc scedulam cartule... Podría tratarse de una fórmula emparentada con FW VII (ed. I. GIL, Miscellanea Wisigothica, Sevilla, 1972), conocida en el scriptorium de Siresa, como puede verse en una donación condal de mediados del siglo IX (UBIETO, CartSiresa, $\mathrm{n}^{\circ} 3$ ).

14. De hecho, es posible que más adelante sí forjaran un diploma a partir de la noticia, pues en un pleito tenido en 1093 con el obispo de Jaca, los entonces canónigos de Siresa adujeron cartam bonam et bene firmatam et ex longinquis temporibus factam, quam fecit quidam episcopus nomine Ferreolus, videlicet ut iam dicte ecclesie quod sibi debebant facere et clericis suis facerent beato Petro de Siresia suisque clericios. Hanc cartam ille episcopus Ferreolus prefatus scripsit et firmavit, cum consilio regis... (A. DURÁN GUDIOL, Colección diplomática de la catedral de Huesca, I, Zaragoza, 1965, no 56). La expresión empleada para referirse a los derechos episcopales es la misma que la de la noticia de 922. Pero cuesta creer que si tal diploma hubiera existido desde esa fecha, no lo habrían copiado en el cartulario, cuyo documento más reciente es de 971.

15. UBIETO, CartSiresa, no 4.

16. Ibid., $n^{\circ} 5$. 
donavit ibi de Scaberri usque ad Siresiam quod debuerunt illi facere faciant ibi. Teste domnus Galindo episcopus... ${ }^{17}$

Los monjes de Siresa fueron perfectamente conscientes de que se trataba de dar una legitimidad nueva a concesiones anteriores. Tan es así que retocaron las dos donaciones condales previas - de ahí que no sepamos la fecha de la segunda-, añadiéndoles un extraño párrafo de inspiración común en el que los condes solicitan al rey Sancho que se constituya en garante de lo que acaban de dar: et ego depreco domno Sancione rege... ${ }^{18} \mathrm{Y}$ de la solemnidad del acto vuelve a dar testimonio una confirmación de 933 debida a García Sánchez I, en la que se recuerda la reunión de rey, conde, obispos y, seguramente con hipérbole, de "todos los señores, de oriente a occidente, que estaban bajo mandato del rey Sancho Garcés" 19 .

No se puede afirmar con seguridad absoluta que actuara un obispo en el entorno condal aragonés antes de esta aparición de Ferriolo. En cualquier caso no podría estar en la cláusula regnante, porque en las dos que se conservan ni siquiera figuran los condes, sólo el emperador Luis o los reyes Carlos, Alfonso y García Íñiguez en 867. En la donación de Galindo Aznar I de este año sí están en juego diezmos y primicias de un área del valle, pero no aparecen ni obispo de Aragón, ni obispo de Pamplona ${ }^{20}$. Lo que, sin ánimo de rizar el rizo, podría indicar que en 922 Ferriolo se limita a confirmar lo que había dado antes el conde. Pero, si los derechos de 922 hubieran sido hasta entonces, aunque fuera nominalmente, del prelado pamplonés, ¿figuraría éste sólo como testigo de la donación? Creemos que en 922 no se instituyen nuevos gobernantes en Aragón, sino que se escenifica en un espacio vinculado al poder condal la tutela de los navarros sobre algo que ya existe. Sólo la convicción de que es decisión navarra la creación de una diócesis aragonesa ha hecho pensar que Ferriolo era el nombre que la rotura del pergamino del códice de Roda hizo desvanecerse. Basta con que el nombre fuera el de su sucesor, quizá el Fortún documentado en 933, ordenado por Galindo de Pamplona como expresión de una jerarquía similar a la que refleja el acto de 922, para que las cosas sean más comprensibles en Aragón y las fechas dejen de resultar incoherentes en Rioja. Pero eso sí, ello implica que la triple ordenación de Galindo no venía necesariamente a ins-

17. Ibid., $\mathrm{n}^{\circ} 6$.

18. Además del sentido general del texto, comparten la muy poco frecuente alusión a Ananías y Safira junto a Judas Iscariote. Alusión que, dicho sea de paso, evoca irresistiblemente el principio de la Regla Común (CAMPOS, J. y ROCA, I., (eds.), Santos Padres Españoles. II. San Leandro, San Isidoro, San Fructuoso. Reglas monásticas de la España visigoda. Los tres libros de las "Sentencias", (Biblioteca de Autores Cristianos: 321) Madrid, 1971, RegCom 2).

19. UBIETO, CartSiresa, $\mathrm{n}^{\circ} 7$.

20. Tampoco en los fondos de San Juan de la Peña aparecen antes de 922 ni el obispo de Pamplona, ni ninguno de Aragón al este de los territorios que después forman parte de la diócesis de Pamplona (UBIETO, A., Cartulario de San Juan de la Peña, Valencia, 1962). 
taurar nuevos obispados, sino también a reconocer e integrar en el reino algunas realidades que ya existían.

Tampoco conviene perder de vista que del texto del Rotense no se desprende que la ordenación de los tres obispos tuviera lugar en una ceremonia única. Es perfectamente posible que la voluntad de encuadrar eclesiásticamente los territorios incorporados en el Pirineo y en el Ebro se tradujera en las ordenaciones episcopales, pero éstas pudieron tener lugar en función de las circunstancias de cada zona. Por ejemplo en Aragón, tras la muerte de Ferriolo. En nuestra opinión, abunda en este mismo sentido la vinculación del obispo de Aragón con el oscuro monasterio de San Adrián de Sasabe. A. Durán Gudiol lo encuentra sorprendente, y sugiere una cierta voluntad navarra de marginar el gran monasterio de Siresa ${ }^{21}$. Una vez más, la premisa de que todo deriva de una decisión del poder pamplonés que crea una diócesis ex novo dificulta la comprensión. Hay un paralelismo esclarecedor en, por ejemplo, el obispado de Valpuesta, vinculado a un monasterio que en el siglo $\mathrm{X}$ en absoluto es comparable a las grandes abadías castellanas y que nace en un contexto local y regional alejado de cualquier diseño político a escala del reino ${ }^{22}$.

El examen del texto de Siresa afloja el nudo que ahogaba la coherencia de la supuesta fecha de la consagración con la toma de Nájera y Albelda, y da pie a plantear una explicación vinculada a la realidad local previa a la llegada de las tropas leonesas y luego navarras. Tomaremos cuatro puntos en consideración.

En el último confín de la Marca Superior de al-Andalus, los valles del Najerilla y el Oja no han contado hasta casi la víspera de la conquista cristiana con centros sólidos de implantación del poder emiral, es decir de mecanismos de encuadramiento territorial y de focos de difusión de nuevas pautas sociales y culturales islámicas ${ }^{23}$. Sólo en tiempos de la fitna del emirato, cuando muchas regiones andalusíes se erizan de fortalezas, aparece en las fuentes el hisn de Nájera ${ }^{24}$, en el mismo tiempo en que también en el tramo inferior del Aragón se fortifican Falces o Caparroso. No más de una generación antes de la conquista

21. DURÁN GUDIOL, A., El monasterio de San Pedro de Siresa, Zaragoza, 1989, p. 13.

22. MARTíN VISO, I., "Organización episcopal y poder entre la Antigüedad tardía y el Medioevo (siglos V-XI): las sedes de Calahorra, Oca y Osma", Iberia. Revista de la Antigüedad 2 (1999) 151-190: p. 176; FERNÁNDEZ CONDE, Religiosidad (cit. n. 8) 352-353.

23. SÉNAC, Ph., "El dominio musulmán: primeras investigaciones", en: Logroño (cit. n. 10) 1933; E. ÁLVAREZ LLOPIS, E., y PEÑA BOCOS, E., "Un espacio de frontera: el Valle medio del Ebro en los siglos VIII a XII", en: VI Estudios de Frontera: Población y poblamiento. Homenaje a Manuel González Jiménez, Jaén, 2006, p. 35-50. Véase la contribución de D. Peterson en este número.

24. Las fuentes árabes relativas a los Banu Qasi, y por ende a la organización musulmana del territorio en la Rioja han sido objeto de profundo análisis por J. LORENZO, a quien agradecemos la posibilidad de consultar su tesis doctoral aún inédita La dawla de los Banu Qasi. Origen, auge y caída de un linaje muladí en la Frontera Superior de al-Andalus, Universidad del País Vasco, 2008. Le seguimos en este apartado. Véase también su contribución a este número de Brocar. 
cristiana por tanto. En períodos anteriores habría que ir hasta el Iregua para encontrar algún centro de este tipo, como el hisn de Viguera o Albelda, fortificada a mediados del IX. Sólo de aquí hacia el sudeste la red adquiere una cierta consistencia: el hisn de Arnedo -probable hogar antiguo de los Banu Qasi-, Calahorra reducida a la condición de hisn, Alfaro y, naturalmente, la madina de Tudela. Cierto que Nájera Ilega a ser calificada de madina en algún texto árabe, pero la impresión que dan los primeros textos cristianos, con sus alusiones aún a la civitas y al suburbium de Tricio -único núcleo junto con Nájera y Viguera calificado de civitas en el siglo $\mathrm{X}^{25}$-, es que su consolidación se deberá a la prolongación de su centralidad bajo la monarquía de Pamplona ${ }^{26}$. La antroponimia también apunta hacia la debilidad tanto de la instalación de población islámica como de la acción de pautas aculturadoras en la Rioja Alta, más allá del valle del Iregua y el entorno más cercano de Nájera ${ }^{27}$.

En los valles del Iregua y el Najerilla, la fuerza simbólica y el discurso de cristianismo militante que emanan de la geografía de las reliquias durante las últimas décadas del emirato son espectaculares. Muy superiores, por ejemplo, a las de todo el reino de Pamplona. En el valle del Iregua, los monjes de Monte Laturce custodian el cuerpo de San Prudencio en un complejo cenobítico consagrado originalmente a San Vicente. El análisis de la transmisión textual de la Vita Prudentii y de todos los testimonios conexos, debidos a N. Igartua, permite tomar en consideración el poco conocido hecho de que una de sus acciones mayores fue la predicación a gentes recién islamizadas o en vías de islamización en el área de Calahorra ${ }^{28}$. A unos quince kilómetros a vista de pájaro hacia al oeste, cerca de Tricio y Nájera, Santa Coloma constituye probablemente la más clara evidencia de lo que estamos diciendo. El martirium en sí pertenece a una familia de edificios altomedievales -San Román de Tobillas, San Vicente del Valle, San Pedro de Arlanza...- vinculada a la acción de personajes o grupos capaces de

25. GARCíA DE CORTÁZAR, J.Á.,"Los comienzos de la organización hispanocristiana del espacio riojano entre los años 923 y 1016", en: Logroño (cit. n. 10) p. 63-92: p. 68.

26. PEÑA BOCOS, E., y ÁlVAREZ LLOPIS, E., "El territorio y el reino de Nájera en la Alta Edad Media", en: B. Arizaga y J.A. Solórzano (eds.), El espacio urbano en la Europa medieval. Encuentros internacionales del Medievo, Nájera 2005, Logroño, 2006, p. 541-587.

27. GARCÍA DE CORTÁZAR, "Comienzos" (cit. n. 25) p. 70.

28. ... ad Civitatem Calagurrensem non longe a flumine Ebro sitam accesit. Ibi multi a fide defecerant, erantque infideles mahomethani, et adorabant, atque venerabantur Doemonem, et mahomethem, et caepit praedicare ibidem atque explicare verbum Dei simul in piis operibus se exercens, et Dominum imitans, qui (ut Evangelium docet) caepit facere, et docere. Tantus itaque erat ardor spiritus, et efficacia verborum ejus, ut omnes viri illius civitatis concurrerint ad audienda verba illius; plurimi vero ad saniorem mentem monitis, et praedicatione Prudentii, et ad tramitem veritatem reducti fuerunt, relictis erroribus, et prava secta, quam antea sequebantur...: IGARTUA, N., Prudencio de Armentia, obispo de Tarazona. Fuentes y contexto histórico de su vida y culto, Vitoria, 2003, p. 59, 117, 165 (texto citado) y passim; ID., "Fuentes hagiográficas referentes a Prudencio de Armentia, obispo de Tarazona", Hispania Sacra 56 (2004) 29-68. 
promover una arquitectura de prestigio, en un amplia área del alto valle del Ebro $^{29}$. Acoge el cuerpo de Santa Coloma, una de las mártires voluntarias de Córdoba de mayor renombre según puede verse por el espacio y el interés que le otorga Eulogio en el Memoriale Sanctorum, y cuyos despojos se salvaron excepcionalmente de la destrucción ${ }^{30}$. Estamos indudablemente en presencia de una comunidad cristiana vigorosa y bien arraigada, cuyos dirigentes mantienen tanto contactos regionales como una relación privilegiada con la mozarabía meridional. Dejan ver una voluntad clara de exhibir uno de los símbolos más poderosos del cristianismo de combate en al-Andalus, así como de emplearlo como mecanismo de cohesión interna. En fin, aproximadamente a otros quince kilómetros hacia poniente está el más célebre de todos y el más antiguo, San Millán, en un santuario semirrupestre rodeado de una aureola de iglesias ${ }^{31}$.

Es sabido que tras la conquista cristiana, uno de los expedientes de que se valieron los nuevos gobernantes para hacerse con rentas y con presencia local fue la confiscación de tierras de musulmanes muertos, huídos o capturados. Algunos ecos de esto son bien conocidos, así la donación por la que en 928 la reina Tota da a Albelda toda la suerte que le había pertenecido en el territorio de Tricio al "pagano Ilamado Abdella iben Mochaoar"32. O la garantía real a San Román de Pampaneto de que disfrutará sin trabas de todo lo que había sido pertenencia de musulmanes ${ }^{33}$. Pero hay otro testimonio que ha sido generalmente alegado en un sentido diferente, y que en nuestra opinión merece examinarse desde esta perspectiva. Se trata del pleito que sostienen en 940 Sancho y Nuño Gómez, seguramente dos hermanos, con su señor en torno al agua del río Tirón que mueve el molino de los primeros ${ }^{34}$. El nudo de la cuestión estribaba en probar si el terreno había sido presura et populatione de cristianos antes de que fuera construído el ingenio. La aparición de la presura ha venido orientando la

29. CABAllero, L., ARCE, F. y UTRERO, M.Á., "Santa María de los Arcos de Tricio (La Rioja), Santa Coloma (La Rioja) y La Asunción de San Vicente del Valle (Burgos). Tres miembros de una familia arquitectónica", Arqueología de la Arquitectura 2 (2003) 81-85. Su carácter minoritario y de prestigio queda confirmado por el examen de una iglesia de este grupo, San Román de Tobillas, dentro de un contexto prerrománico alavés mucho más amplio: SÁNCHEZ ZUFIAURRE, L., Técnicas constructivas medievales. Nuevos documentos arqueológicos para el conocimiento de la Alta Edad Media en Álava, Vitoria, 2007.

30. EULOGIUS, Memoriale Sanctorum, III, 10 (ed. I. GIL, Corpus Scriptorum Muzarabicorum, Madrid, 1973).

31. UBIETO, A., Cartulario de San Millán de la Cogolla, Valencia, 1976, nº 77 y 78.

32. UBIETO, A., Cartulario de Albelda, Valencia, 1960, $\mathrm{n}^{\circ} 5$.

33. Ibid., $\mathrm{n}^{\circ} 8$.

34. ... in illis diebus habuerunt Sancho Gomiz et Nunnu Gomiz iudicium cum donno super aqua de molino. Et mandavit legem et libro ludicum ad Sancio Gomiz et ad Nunnu Gomiz firmare ex contra donnum qui sic fuit ipso molino de Nunnu Gomiz in presura de christianos et populatione de christianos ante fuit posito in flumine de Tirone. Et aduxerunt Sancio Gomiz et Nunnu Gomiz XII testimonias et tres fiadores... : UBIETO, SMC I, nº 27. 
interpretación de esto en el sentido del asentamiento de gentes venidas del norte o del oeste. Pero habría de tenerse en cuenta que la presura está bien atestiguada como derecho vinculado a la vecindad que se ejerce en el término de una aldea, dentro de unos usos comunitarios ${ }^{35}$. Pensamos que lo que el señor sostenía frente a los Gómez era que el terreno había sido apropiado antes por musulmanes, lo que implicaba para él algún tipo de derecho sobre la tierra y lo que en ella se encontraba. Esto significaría que no se limitaron los dirigentes cristianos a confiscar lo que había quedado sin dueño, sino también a imponer rentas o derechos sobre todo aquello que había estado o estaba en manos musulmanas. Ahora bien, nos interesa especialmente comprobar cómo ganan el juicio los dos hermanos: aportan doce testigos y tres fiadores que prestan juramento, cosa que los jueces consideran suficiente para sentenciar a su favor. En otras palabras, en un medio donde había presencia musulmana, el tejido comunitario compuesto por familias cristianas permanecía denso y bien implantado en el territorio, puesto que no hay problema después para encontrar un número elevado de vecinos cuyo testimonio es válido y que estaban por ende instalados cuando tuvieron lugar los hechos a averiguar ${ }^{36}$.

Entre los años 1014 y 1020, piezas importantes del área de Tobía son sometidas por Sancho el Mayor a San Millán de la Cogolla. El 24 de junio del primer año son cedidas a la abadía las villas de Ledesma y Colia (hoy Villaverde de Rioja), en los extremos oriental y occidental del valle respectivamente, así como los monasterios de San Cristóbal y San Pedro. De este último hay otro instrumento del mismo año con el detalle de su patrimonio, cosa que también poseemos para San

35. ... equalem cum Soto longo et Navas et in illa via de Vallego, ita ut in pressuras et ligna cedenda omnibus locis cum villas predictas equale censura genua et libera... (Ibid., $\mathrm{n}^{\circ} 123$ ); ... in circuitu eius ecclesie [San Sebastián de Artable] rusticos ad populandum aggregare, domos hedificare, vineas et arbores vel ortos plantare, exitus et regressus habere, presuras habere cum suis vicinis... : LEDESMA, M.L., Cartulario de San Millán de la Cogolla (1076-1200), Zaragoza, 1989, no 376bis.

36. Estos dos hermanos aparecen también en un juicio anterior, de 936 (UBIETO, SMC I, $\mathrm{n}^{\circ}$ 23) que pierden porque no pueden aportar testigos que apoyen su derecho sobre un campo. Ellos alegan que lo poseían desde el tiempo en que Abelmondar Téllez pobló esa tierra. Creemos con J.A. García de Cortázar que tal campo formaba parte de un terrazgo de la comunidad instalada allí antes de la llegada de los dirigentes cristianos. Pero discrepamos en que de la lectura de los dos juicios se desprenda que no todos los vecinos estaban de acuerdo en que la presura fuera un título justo de posesión de la tierra o que los cristianos del texto de 940 sean los que Ilegan con Abelmondar Téllez. Los hermanos Gómez pierden el juicio de 936 porque no encuentran quién testifique a su favor y el de 940 lo ganan porque sí lo hacen. Precisamente ganan el que dirime la autoría de una presura, pues en el primero no se habla de tal cosa. Eso sí, la presura sólo puede venir legitimada por la comunidad de vecinos. Y no vemos cómo pueden vincularse los cristianos a que se alude en 940 con los acompañantes de Abelmondar (GARCÍA DE CORTÁZAR, "Comienzos" (cit. n. 25) 76). 
Cristóbal gracias al documento de confirmación de 102037. De los dos, San Pedro, situado en Villanueva, que a su vez comparte vecindad con Matute, es el más modesto. Además de derechos de uso del monte comunes con Tobía, Matute y Villanueva, tiene un número indefinido de parcelas -sólo se precisan ocho-, cuatro viñas y un molino y medio. Eso sí, dispersas por toda la zona, desde los alrededores del castillo hasta Bovadilla o Ledesma. Por su parte, San Cristóbal se dice situado junto al castillo de Tobía, pero en término de Tobía y Matute. Participa como San Pedro en leñas, aguas y pastos y disfruta también de presencia en todo el territorio -Colia, Baños, Villanueva, Matute...-, pero su patrimonio es mayor y presenta algún rasgo de mucho interés. En 1020 se cuentan nueve campos, dieciséis viñas, dos molinos, diez sernas, un huerto y cuatro arboledas. Varios de los topónimos menores donde tiene tierras son hagiotopónimos, como Santa Eugenia, Santa Eulalia -donde efectivamente hay una iglesia ${ }^{38}$ - o San Martín -este último posesión del monasterio. En ocho casos, se recuerda de quién había sido la viña, la serna o el manzanal antes de integrar el patrimonio de San Cristóbal. De estos anteriores propietarios, tres simplemente son calificados de dompno, uno lo es de magister, tres de Mer, y uno de obispo. En efecto, se menciona la serna de dompno Sesuto episcopo, y acto seguido la viña que fue de Mer Sesuto. Si como parece probable es la misma persona, podría tratarse del Sesuldo obispo de Calahorra que consagra Galindo de Pamplona y que ha dejado alguna huella en la documentación albeldense ${ }^{39}$. Y mostraría una relación entre el cargo eclesiástico y el enigmático Mer (¿un apócope de magister?). Este ¿título? no es exclusivo de Tobía. En Añana Mer Nuño y Mer Galindo son los representanes de San Millán en los años 30 y 40 del siglo $X^{40}$. Otros Mer aparecen por los años de la cesión de San Cristóbal de Tobía, ora como titulares de tierras limítrofes de San Millán, ora vinculados a pequeñas comunidades y monasterios ${ }^{41}$. Pero fuera de Tobía no se encuentran tres juntos en un mismo contexto local y documental. Cosa que se confirma más tarde, en una descripción del patrimonio fundiario de Santa María de Nájera compuesta al parecer en el siglo $\mathrm{XI}^{42}$. De las docenas de nombres recogidos en numerosas localidades de la comarca, sólo encontramos un Merdaniel en Villamezquina, frente a cinco Mer (García, Aznar, Albeto, Jimeno y Dulquito) en la zona de Tobía. Qué signifique esta peculiaridad es difícil de decir, pero todo

37. Ibid., $\mathrm{n}^{\circ} 151,152,153$ y 171.

38. RODRÍGUEZ DE LAMA, I., Colección diplomática medieval de la Rioja (923-1225), Logroño, 1976, nº 14.

39. UBIETO, CAlbelda, $\mathrm{n}^{\circ} 2$ y 3.

40. UBIETO, SMC I, no 21 y 50.

41. Ibid., $\mathrm{n}^{\circ}$ 176, 178, 182, 303 (aquí se transcribió Men Sancio en una copia del siglo XVI, es probable que pusiera Mer). Igualmente, el Merdidato propietario de tierras limítrofes de las de Albelda en Jubera podría ser una trasnscripción incorrecta de Mer Didaco (UBIETO, CAlbelda, $\mathrm{n}^{\circ}$ 64).

42. RODRÍGUEZ DE LAMA, CDRioja, $\mathrm{n}^{\circ} 14$. 
parece apuntar a que, en una zona de intensa y estructurada vida eclesiástica, el monasterio de San Cristóbal, a los pies de un castillo ${ }^{43}$, está plenamente integrado en el tejido social y territorial circundante, si es que no constituye en sí mismo un importante mecanismo vertebrador.

Visto todo esto, creemos que es más coherente con todas la fuentes disponibles y con el contexto histórico general y local, sostener que la consagración de un obispo de Tobía es la fórmula que emplea el nuevo poder cristiano para integrar una iglesia local vigorosa, estructurada y bien arraigada. Nada tiene de sorprendente que en este período aparezca un obispo en una zona rural sin antecedentes en la geografía diocesana tardoantigua. Dejando de lado que la problemática de obispos constituido in villiolis, in vicis, o in agris (entiéndase distritos rurales) es fenómeno conocido en Occidente $^{44}$ o que hay indicios de nacimiento de sedes poco conocidas en la última fase del reino visigodo, entre ellas la de Alesanco ${ }^{45}$, la existencia de obispos sin diócesis cuya suerte depende de su articulación o no con el poder político ha sido bien estudiada ${ }^{46}$. En nuestro caso, no es extraño que la dignidad episcopal pasara pronto a Nájera, una vez confirmada su elección como centro del poder político, ahora cristiano, en la región. Es difícil sin embargo precisar más: el obispo Teodorico figura en un diploma manipulado relativo a Santa Coloma, sin indicación de sede ${ }^{47}$. En fin, como última observación, Tobía no tiene por qué designar un espacio diocesano, como piensa E. Sainz Ripa ${ }^{48}$. Exactamente igual que Sisabensis en Aragón, Tobiensis se refiere seguramente al monasterio donde tiene su sede el obispo. En nuestra opinión, a San Cristóbal de Tobía.

\section{Comunidades y territorio}

La constatación de la presencia de comunidades estructuradas y bien arraigadas antes de la llegada de navarros y leoneses confiere una profundidad notable al estudio de las formas de apropiación del territorio en esta zona: abre un

43. Recuerda a la pareja Santa María de Irache - castillo de San Esteban que nuclean el territorio de Deio, en la actual Tierra Estella, también conquistado por los navarros poco antes que Nájera y Viguera.

44. Así, por limitarnos a dos referencias bien conocidas, la encontramos en el c. 19 de la Admonitio generalis de 789 y en el 22 del sínodo de Frankfurt de 794: BORETIUS, A., (ed.), $\mathrm{MGH}$, Capitularia Regum Francorum, I.

45. SÁNCHEZ ALBORNOZ, C., "Fuentes para el estudio de las divisiones eclesiásticas visigodas", en: C. Sánchez Albornoz, Investigaciones y documentos sobre las instituciones hispanas, Santiago de Chile, 1970, p. 66-106; ID., "Sobre las Nominae Sedium Episcopalium visigodas", en: Ibid., p. 108-113; VÁZQUEZ DE PARGA, L., La división de Wamba. Contribución al estudio de la historia y geografía eclesiásticas de la Edad Media española, Madrid, 1943.

46. MARTíN VISO, "Organización episcopal" (cit. n. 22).

47. RODRÍGUEZ DE LAMA, CDRioja, nº 1.

48. SAINZ RIPA, Sedes (cit. n. 6) p. 173. 
portillo hacia períodos anteriores y desvela mecanismos de articulación con el poder político que operan en tiempos muy próximos a los testimonios escritos, y no en un vago y conjetural pasado como ocurre en otras regiones. Se aprecia un armazón territorial en el que actúan, manipulan y se introducen poderosos venidos tras la conquista o gentes e instituciones locales que progresan a la sombra de los nuevos dueños del país. Naturalmente, son estas acciones las que nos permiten penetrar en tal armazón, en la medida en que generan derechos y pleitos, es decir textos. Por otro lado, la documentación de la Rioja Alta es particularmente rica en informaciones, casuística, excepciones y conflictos en torno a la explotación del territorio, a lo que se añade una producción historiográfica de primer orden que facilita grandemente aproximaciones parciales como la nuestra ${ }^{49}$.

La apropiación del territorio se materializa a través del juego de dos tipos de espacios. El primero es el que se organiza en torno a los núcleos de población y comprende, desde el punto de vista del paisaje, casas y solares, eras y huertos, campos y viñas, molinos y prados... Como definición, puede decirse que es el espacio donde es posible la apropiación individual, permanente o temporal, de un terreno delimitado, cosa que a su vez se organiza en función de la oposición entre intus -el solar que contiene la casa y cuya posesión da derecho a la vecindad- y foras -el conjunto del terrazgo donde se ejercita esa vecindad dentro de una lógica comunitaria ${ }^{50}$. Aquí la serna es omnipresente. Sus menciones se cuentan por docenas, si no por algunas centenas en la documentación emilianense. No es nuestro objeto abordar específicamente la cuestión de las sernas $^{51}$; sólo nos interesa subrayar su carácter multiforme. En efecto, hay sernas recién roturadas ${ }^{52}$, sernas parcialmente cultivadas $^{53}$, sernas con huertos, viñas, árboles, sotos y linares ${ }^{54}$, y aun sernas habitadas. Desde otro punto de vista, hay

49. Pensamos naturalmente en primer lugar en los numerosos trabajos que J.A. García de Cortázar ha dedicado a esta tierra durante cuatro décadas y que han sido sintetizados por el autor en su aportación a la obra colectiva Historia de la ciudad de Logroño, abundantemente citada en estas páginas.

50. Sobre este juego de conceptos: LARREA, J.J., "Cadres de vie en Espagne chrétienne", en: P. Bonnassie y P. Toubert (eds.), Hommes et sociétés dans l'Europe de l'An Mil, Toulouse, 2004, p. 137-162; ID., "De la invisibilidad historiográfica a la apropiación del territorio: aldeas y comunidades en la España cristiana (siglos X y XI)", en: J.I. de la Iglesia Duarte (ed.), Cristiandad e islam en la Edad Media Hispana. XVIII Semana de Estudios Medievales de Nájera, Logroño, 2008, p. 169-207.

51. BOTELLA, E., La serna: ocupación, organización y explotación del espacio en la Edad Media (800-1250), Santander, 1984; GARCÍA DE CORTÁZAR, J.Á., "La serna, una etapa en el proceso de ocupación y explotación del espacio", en: En la España Medieval (Estudios dedicados al profesor D. Julio González González) 1 (1980) 115-128.

52. LEDESMA, SMC II, n $\mathrm{n}^{\circ} 335 \mathrm{bis}$. En esta nota y en las que siguen nos limitamos a consignar algunos ejemplos a título meramente ilustrativo.

53. UBIETO, SMC I, n 115.

54. Ibid., $\mathrm{n}^{\circ} 364 ; 340$; LEDESMA, SMC II, $\mathrm{n}^{\circ} 487$. 
sernas del rey ${ }^{55}$ y de los magnates ${ }^{56}$, de los monasterios grandes y pequeños ${ }^{57}$, de laicos de fortuna diversa ${ }^{58}$, de presbíteros ${ }^{59}$, de comunidades de $\operatorname{vecinos}^{60} \ldots$ La serna recorre prácticamente todo el espectro social. En cuanto a su función, es uno de los mejores indicadores de la fluidez de este espacio de casas y campos. Basta poner en una perspectiva diacrónica el abanico de usos agrícolas, desde el desbroce hasta el viñedo, para intuir que la serna ha sido un mecanismo clave en la movilidad altomedieval de los terrazgos. Es sabido que en general estos desplazamientos se van ralentizando a partir de finales del siglo VIII, pero no es menos cierto que suelen conservarse trazas de ellos y de los sistemas que los han vehiculado hasta más tarde. No pretendemos pues que hábitat y terrazgos se muestren igual de fluidos en torno al año Mil que en torno al 700, naturalmente. Pero sí que el paisaje sigue presentando una cierta flexibilidad. Si tenemos en cuenta que además las sernas pueden generar solares, el abánico de movilidad va desde el terreno inculto hasta el barrio o la aldea. Así ocurre cuando el rey Sancho de Peñalén da San Millán una serna donde nuevos pobladores podrán construirse casas con derecho de vecindad en el término ${ }^{61}$. O cuando su madre la reina Estefanía instala en una serna de Sojuela a ciertos fugitivos, también con derecho en la comunidad de pastos de los pueblos circundantes, las llamadas villas del $\mathrm{Campo}^{62}$.

Junto a la serna, hay otras maneras de generar nuevos núcleos de hábitat. Los terrenos adyacentes a las iglesias, los dextros, también pueden convertirse en solares. Así en Artable el circuito de 62 pasos en torno a la iglesia de San Sebastián podrá acoger colonos con acceso al término de la aldea y con derecho a presura con el resto de los vecinos ${ }^{63}$. En otras ocasiones ignoramos la fórmula, pero encontramos a algún magnate instalando pobladores ${ }^{64}$. De mucho interés es el caso de la repoblación del monasterio abandonado de San Andrés de Jubera, donde se combina la iniciativa señorial y el consenso comunitario, cabe suponer que tras una negociación que desconocemos: hacia 1062, el obispo Gomesano encarga a un don García la instalación de

55. UBIETO, SMC I, nº 184.

56. Ibid., $\mathrm{n}^{\circ} 90$.

57. Ibid., $\mathrm{n}^{\circ}$ 2, 6, 12, 303; LEDESMA, SMC II, $\mathrm{n}^{\circ} 335$ bis.

58. UBIETO, SMC I, n' 102 y 130.

59. Ibid., $\mathrm{n}^{\circ} 140$.

60. Ibid., $\mathrm{n}^{\circ} 184$.

61. Ibid., $\mathrm{n}^{\circ} 401$.

62. RODRÍGUEZ DE LAMA, CDRioja, $\mathrm{n}^{\circ} 18$.

63. LEDESMA, SMC II, $\mathrm{n}^{\circ} 376 \mathrm{bis}$.

64. Tal y como se disponen los testigos, da la impresión de que los promotores de la instalación de colonos en San Anacleto no es sólo obra de García de Nájera y de uno de sus barones, Jimeno Garcés, sino también de los otros tres que figuran a continuación, cada uno "con todos sus vecinos": UBIETO, CAlbelda, $\mathrm{n}^{\circ} 49$. 
nuevos pobladores a los que se ofrecen garantías sobre sus bienes y el concejo de Jubera segrega una parte de su término para adjudicárselo a San Andrés ${ }^{65}$. Esta capacidad comunitaria nos Ileva hasta aquellos barrios o aldeas que parecen surgidos al margen de iniciativas de poderosos, dentro de la dinámica de movilidad de terrazgos y hábitats que hemos entrevisto. Más abajo examinaremos algún ejemplo.

El segundo espacio corresponde a los montes, las aguas y los pastos. Está orgánicamente vinculado al anterior, en la medida en que su acceso depende de la vecindad en uno u otro núcleo. Pero este espacio, donde no es posible la apropiación individual, se rige por un sistema complejo en el que se superponen de forma diversa en el tiempo y en el espacio los distintos niveles de derecho que corresponden a cada comunidad. Y está sometido a un control mutuo constante entre sus usuarios. La gradación de niveles de uso recorre una escala en cuyo extremo superior aparece la estancia día y noche del ganado en los pastos, lo que implica el derecho a construir cabañas. Más abajo está el aprovechamiento sólo diurno, debiendo ser retirados los rebaños al atardecer. Y aún hay quien tiene vetado todo acceso y ha de conformarse con alimentar unas pocas cabezas en los barbechos y ribazos del entorno de la aldea. Ésta es una circunstancia poco documentada, pero muy interesante. Se encuentra en uno de los pocos diplomas originales de San Millán ${ }^{66}$. En 1013 un barón da su monasterio de Santa María, sito junto a Villar de Torre, a la abadía. Como en otras donaciones similares, se indica que comparte pastos, montes y aguas con los hombres de Villar de Torre y se le declara exento de participar en tributos, homicidios y otras causas que pudieran recaer sobre la comunidad de vecinos. Ahora bien, cuando se especifica en qué consiste tal participación en los pastos, se aclara que los ganados del monasterio no tienen acceso al vedado de los vecinos, sino que han de pastar entre viñas y parcelas, sin dañar los cultivos. El interés del testimonio es doble. Primero, porque hace luz sobre situaciones de inferioridad que pueden repetirse en otro lugares y que son camufladas por la alusión genérica al pasto común con los vecinos. Segundo, porque muestra que la creación de un núcleo de hábitat no tiene repercusión automática en el espacio de pastos y montes, sino que viene filtrada por el juego de fuerzas local.

La combinación de derechos de uso y espacios traza una geografía compleja, jerarquizada y, quizá paradójicamente, más rígida que la del espacio de hábitats y terrazgos. En la comunidad de pastos de Tobía, los de Ledesma han de abandonar los pastos al atardecer y volver al término de la aldea, mientras que los de Colia pueden seguir allí -cosa que se traslada luego a San Millán

65. Ibid., $n^{\circ} 44$ y 45.

66. UBIETO, SMC I, $\mathrm{n}^{\circ} 146$. 
cuando estas villas le son cedidas en $1014^{67}$. En las dehesas sobre las que ejerce un derecho superior la comunidad de Madriz, los de Villagonzalo tienen en algunas derecho de pasto, pero no de leña, en otras ni uno ni otro; el pasto en algunas es durante el día, en otras pueden hacer cabañas junto con los hombres de Cordovín y Terrero ${ }^{68}$. La transgresión de la norma de cada vedado, de cada dehesa y de cada majada da lugar a sanciones, para las que se conservan algunas tarifas. En éstas, lógicamente, se repite el tipo de falta, pero el montante varía en función de quién y cómo la fija. La protección del coto de San Millán establece el prendimiento de una cabeza de cada cinco más sesenta sueldos si se erigen cabañas; si sólo se entra para pastar, se pierde una de cada diez en el ganado menor y un sueldo por cabeza de ganado mayor. Por su parte, la tala de árboles arraigados se castiga con 60 sueldos; el de ramaje con cuatro argénteos más la pérdida de la herramienta ${ }^{69}$. En cambio, los usos de Villarejo establecen sólo cinco sueldos por incisión en árbol, si bien la introducción furtiva de piaras también se paga con una cabeza de cada diez ${ }^{70}$. Naturalmente, todo esto implica la presencia de guardas y sobre todo, del control mutuo entre los usuarios.

Es en este ámbito donde algunas comunidades exhiben músculo. En el valle del Cárdenas, la comunidad de Madriz muestra durante mucho tiempo un enérgico control del territorio con el que el mismo San Millán apenas puede competir. A pesar incluso de que durante casi siete años, seguramente con Sancho de Peñalén, la tenencia de Madriz fue encomendada a la abadía ${ }^{71}$. Una serie de litigios y pactos con las comunidades del entorno y con el monasterio evidencia el dinamismo de esta comunidad. En 1050 es el rey quien tiene que poner fin al enfrentamiento que, según el redactor, venía instigando el diablo entre los monjes y la "vecina plebe de Madriz". Delimita un término adyacente al primer coto de San Millán para su uso exclusivo y manda que en adelante sus ganados gocen de derechos de pasto y leña por todo el territorio de Madriz ${ }^{72}$. Sin embargo, no sólo no es evidente que el derecho vaya más allá del pasto diurno, sino que la abadía quedaba aún lejos de tener el mismo acceso que los hombres de Madriz. Prueba de ello es que es el concejo el que sigue controlando yermos y rozas, de modo que en 1082 don Muño de San Millán se ve obligado a comprar a los hombres de Madriz terrenos para roturar y hacer novales que luego cederá al monasterio ${ }^{73}$. Y al parecer, tres años después la comunidad vuelve a

67. Ibid., $\mathrm{n}^{\circ} 151$ y 152

68. Ibid., $\mathrm{n}^{\circ} 231$.

69. Ibid., p. 78.

70. Ibid., $\mathrm{n}^{\circ} 392$.

71. Ibid., $\mathrm{n}^{\circ} 231$.

72. GARCÍA DE CORTÁZAR, J.Á., El dominio del monasterio de San Millán de la Cogolla (siglos X a XIII). Introducción a la historia rural de Castilla altomedieval, Salamanca, 1969, p. 214-215.

73. LEDESMA, SMC II, n 50. 
hacérselo pagar a los monjes cuando pasa directamente a San Millán ${ }^{74}$ En 1137 toda la villa de Madriz es transferida al señorío emilianense ${ }^{75}$, lo que no les impide en absoluto pleitear en 1155 por una dehesa con los de Villar de Torre en un juicio muy interesante por más de un motivo. Primero por el escenario mismo, ya que la asamblea judicial se desarrolla en primera instancia en la misma dehesa y sólo se traslada a una iglesia para prestar los juramentos. Y segundo porque para ganar, los de Madriz han de obtener el testimonio favorable de doce hombres de dos aldeas cercanas, Villarejo y Villadolquit, y además asegurar por juramento que esas dos aldeas no tienen comunidad alguna de término o pastos con ellos ${ }^{76}$. En ese mismo año establecen, sin intervención de autoridad superior alguna, un acuerdo para compartir pasto diurno con otra comunidad importante, la de Pazuengos, en varios espacios del fondo del valle ${ }^{77}$. En 1184 pierden en duelo judicial tres majadas a favor de Villagonzalo ${ }^{78}$.

San Millán se las tiene que ver también con los de Pazuengos, valle arriba, y con los de Ojacastro, al otro lado de la sierra. Pazuengos evidencia en primer lugar la distancia que va de la donación solemne al uso real del territorio. En 944 Fernán González da a San Millán el monasterio de Santa María y toda la villa de Pazuengos con circuitos, montes, dehesas y pastos ${ }^{79}$. Mucho después, en 1070, los de Pazuengos destruyeron la cabaña de los monjes y expulsaron sus vacas de una vaqueriza. El pleito subsiguiente lo gana San Millán sin problema, pero el hecho en sí muestra que la entrada de los ganados de la abadía en los términos de Pazuengos no tuvo lugar como parece desprenderse de la solemne donación condal, sino más bien a través de un reparto preciso de aprovechamientos y espacios. De otro modo, el mero hecho de hallarse en término de Pazuengos habría sido argumento bastante, siendo así que lo que el rey sentencia es que esa majada en concreto era de San Millán. Por otro lado, el documento matiza la imagen monolítica que a veces puede desprenderse de la acción de las comunidades. Relata cómo dos vecinos de Pazuengos juran a favor de San Millán, reconociendo que habían actuado ilegítimamente, y cómo esto da lugar a un conflicto entre las gentes del pueblo ${ }^{80}$.

74. Ibid., $\mathrm{n}^{\circ} 96$.

75. Ibid., $\mathrm{n}^{\circ} 370$.

76. Ibid., $\mathrm{n}^{\circ} 395$.

77. Ibid., $n^{\circ} 396$.

78. Ibid., $\mathrm{n}^{\circ} 442$.

79. UBIETO, SMC I, no 35.

80. Inter hec fuit altercacio inter homines de Pazongis. Quibusdam ex illis dixerunt ad suos "per vim ibimus ad illo ganato, quia scimus ut ganato de Sancti Emiliani in Losellas debet sedere": Ibid., $\mathrm{n}^{\circ}$ 384. No es difícil imaginar detrás de esta actitud enfrentamientos entre vecinos, de tal calado que para algunos parece resultar rentable jugar a la alianza con el monasterio. De hecho, sería un error representar el funcionamiento interno de estas comunidades como algo libre de las duras, a veces sórdidas y siempre complejas líneas de conflicto que tensan la convivencia y hacen evolucionar la relación con el territorio de otras comunidades similares 
En cuanto al concejo del valle de Ojacastro, un pacto hecho en 1063 entre éste y San Millán tiene la virtud de sacar a la luz una faceta de mucha enjundia en las relaciones entre la abadía y las comunidades circundantes, que de otro modo parecen presididas siempre por la competencia y el conflicto ${ }^{81}$. En efecto, abad y valle acuerdan cómo deberán reparar los de Ojacastro el daño hecho en caso de que por hambruna o cualquier penuria deshicieran las cabañas de ovejas y vacas de los monjes. Pero acto seguido se evocan otros vínculos consuetudinarios que no se perciben como incompatibles con el conflicto. Se diría más bien que son la otra cara de la moneda en un sistema de relaciones a varios niveles. Así, el refugio en el monasterio del vecino de Ojacastro acusado de homicidio o las que parecen romerías de las gentes del valle con intercambio de vino para los vecinos y votos de quesos y dinero para la abadía. Y sobre todo, algo de una potencia simbólica notabilísima: la custodia del cuerpo de San Millán, de la que se dice que solían ocuparse los de Ojacastro. Se establece en efecto que si por alguna emergencia el cuerpo ha de volver a llevarse de Yuso a Suso, la guardia en el traslado se encomendará prioritariamente a los hombres de Ojacastro ${ }^{82}$. Es un destello de información sobre los vínculos que traban estas reliquias de primer orden con la sociedad circundante $-y$, sospechamos, sobre el malestar que pudo generar la apropiación simbólica de San Millán por el rey García y su flamante monasterio de Yuso.

En torno a un San Millán de presencia e influencia creciente, Ojacastro, Pazuengos y Madriz se muestran como los puntos centrales de la organización de este espacio de montes. En torno a ellos, en particular en torno a Madriz, gravitan otras comunidades menores, como Villagonzalo, Villar de Torre o Cordovín, que ocupan una posición secundaria en la jerarquía de aprovechamientos. Hacia el este se encuentran la comunidad de Tobía, por la que nos interesaremos inmediatamente y la de las villas del Campo -Fuenmayor, Entrena, Medrano, Villiela, Corcuetos (Navarrete) y Hornos de Moncalvillo- que se extiende desde las orillas del Ebro hasta la sierra y aparece con nitidez en 106083. Entre una y otra está el área de Santa Coloma, recostada en la sierra, para la que no poseemos sino muy escasa información.

en períodos algo más recientes: VIADER, R., "Maisons et communautés dans les sociétés montagnardes. Le temps juridique (XIII"-XVe siècle)", en: Montagnes médiévales, Paris, 2004, p. 263-291.

81. LEDESMA, SMC II, n 287bis. En la edición se le atribuye por error la fecha de 1053. J.Á. García de Cortázar entiende que el texto debe ser posterior en un siglo a la fecha que exhibe. Ignoramos por qué : GARCÍA DE CORTÁZAR, J.Á., "Castellanización y feudalización del espacio riojano entre 1076 y 1116. El nacimiento de Logroño", en: Logroño (cit. n. 10) p. 131-168: p. 149.

82. Consueverunt et corpus beati Emiliani servetur ab illis in altari cum causa venerint vigilandi. Et si forte aliqua necesitate acciderit quod corpus beatissimi Emiliani deferatur ad superiorem locum ubi prius sepultus fuit nisi prius Oiacastrensi conveniant alicui non reddantur et illis custodiant corpus in vigiliis in eundo et redeundo (ref. nota anterior).

83. RODRÍGUEZ DE LAMA, CDRioja, $\mathrm{n}^{\circ} 18$. 


\section{Homicidio pro termino}

La comunidad de Tobía explota el valle que forma el arroyo del mismo nombre desde su nacimiento en la sierra de San Lorenzo hasta la desembocadura en el Najerilla. Los núcleos dominantes son los que se sitúan a la entrada del valle, Tobía y Matute. Ahí se encuentran el castillo y el monasterio de San Cristóbal; ambos pueblos figuran como sedes de tenencias en el siglo $\mathrm{XI}^{84}$. En una vaguada a medio camino entre Matute y San Millán se encuentra Colia, posiblemente un núcleo surgido más tarde del que se dice en 1014 que está situado in confinio villarum Tubie et Matute. Cuando en esa fecha Ledesma y Colia son cedidas a San Millán ${ }^{85}$, se recoge el testimonio de ancianos en relación a los usos en el aprovechamiento de pastos: ...cum omnibus pertinentiis vel que a veteris fuerint testificata senibus, ita ut cum villis communia habere pascua... ${ }^{86}$ Es elocuente notar que aquella que está en el confín de Tobía y Matute comparte plenos derechos, mientras que Ledesma, de la que no se dice tal cosa, sólo goza de pasto diurno, debiendo volver a sus términos al anochecer. Sin embargo, las relaciones con Colia - detrás de la cual estaba San Millán- fueron tormentosas, y en algún momento del siglo XI los de Tobía y Matute expulsaron de su vecindad a Colia, por la fuerza y con mucha tenacidad:

Notum sit omnibus hominibus quod Villula Colia barrio fuit de Tuvia et vicinitatem habuit tam in erbis quam in montibus et terminis simil cum Matute. Transactis itaque multis annis contigit ut illos de Matute et Tuvia proiecerunt de vicinitate in montibus et erbis et terminis ad illos de Collia, multumque laborantibus pro temporibus et annis, usque venerunt ad campeatura $^{87}$.

Obsérvese una vez más la relación jerárquica, pues no se habla de división, sino de expulsión. Y nótese también cómo un conflicto puede transformar un barrio en una aldea, lo que nos reenvía a la noción de plasticidad de este espacio que mentábamos más arriba. En cualquier caso, la ruptura implicaba que Colia quedaba reducida al mismo nivel de aprovechamientos que Ledesma, es decir a la imposibilidad de poner cabañas en el monte. La reacción de Colia, apoyada sin duda por el cenobio, fue la de ir a juicio en 1097 para sostener su derecho a poner cabañas en la margen izquierda del arroyo, como venía haciendo. El juicio se solventó en duelo y el de Colia venció al representante de Tobía y Matute:

84. FERNÁNDEZ DE LA PRADILLA, Ma.C., "La articulación del poder pamplonés en el espacio riojano" , en: Logroño (cit. n. 10) p. 35-46: p. 42-43; PEÑA BOCOS, E., "Alfoces y tenencias: la Rioja", en: J.Á. García de Cortázar (ed.), Del Cantábrico al Duero. Trece estudios sobre organización social del espacio en los s. VIII a XIII, Santander, 1999, p. 376-411.

85. UBIETO, SMC I, n' 151 y 152.

86. Ibid., $\mathrm{n}^{\mathrm{o}} 152$.

87. LEDESMA, SMC $I I, \mathrm{n}^{\circ} 266$. 
Et dixit ita viro quia vos de Colia de die debetis pascere et nocte a domos vestras revertere et cabanna in montibus non debetis ponere. Et ille aliud Blasco Sarrazin de Colia iurans dixit "absit. Sed de illo ruivo de Tuvia unde nascet usque ad Naiela flumen vicinos sumus in montibus et erbis et defesis de ista parte de aqua, et ponemus cabannas nostras latus aqua ex nostra parte, et pascimus per omnes montes et nocte revertimur ad cabannas". Et fuit litis in campastro, et fuit victus et superatus illo de Matute...

Este pleito nos sirve para encuadrar una investigación llevada a cabo algo antes, en 1089, por orden del conde García Ordóñez. Habiendo sido muerto un hombre en término de Matute, se trataba de saber si Colia era o no barrio de Tobía y debía por ende pagar el homicidio junto con Tobía y Matute. Parece claro que para este año los de estos dos pueblos ya sostenían de palabra y de hecho, acosando y cogiendo ganado de los de Colia, que estos últimos no formaban vecindad con ellos. Los testigos reunidos por los tres enviados del conde de entre los hombres de varias aldeas de la zona prueban que Colia era en efecto barrio de Tobía y que debían pagar conjuntamente el homicidio:

... prescrutare illa villa Cogga si debebat dare homicidio cum Matute et Tuvia pro illo homine qui fuit mortuus in termino de Matute. Et isti probatores, qui ante superius diximus, et de Matute fuerunt (...) Et de Anguidanos (...) Et de Villanova (...) Et de Matrice (...) Isti omnes probaverunt et testificaverunt quod Collia barrio erat de Tuvia et in solo homicidio debebant dare cum illis et guarda tenebant cum illis, et similiter comune habere in defesa et in erbas et terminos pascere et iacere cum illis ${ }^{88}$.

Lo curioso del caso es que San Millán se siente vencedor en un pleito del que resulta que sus hombres habrán de pechar. Parece que son ellos los instigadores del juicio según el tenor de las primeras frases; consignan los nombres y cargos de los representantes de la abadía que asistieron a la sentencia; acaban recordando una serie de altercados con intervención del merino que contribuyen a probar la vecindad de los de Colia con los de Tobía. No es la única ocasión en que San Millán maniobra de esta manera. En 1076, fue a través del pago al rey de 200 sueldos por el homicidio de un hombre muerto en unos molinos cuyo terreno estaba en disputa entre Terrero -villa emilianense- y Alesanco, como San Millán se aseguró la titularidad de un terreno que hasta entonces tenían los de Alesanco ${ }^{89}$. En Colia, ¿qué gana San Millán? La respuesta está en el vínculo orgánico entre el homicidio y el aprovechamiento de los pastos que aparece con nitidez en el pasaje reproducido más arriba.

Este vínculo entre autoridad judicial del rey sobre las comunidades y acceso a los pastos nos interesa especialmente. Obsérvese, por cierto, que el homicidio

88. Ibid., $\mathrm{n}^{\circ} 189$.

89. UBIETO, SMC I, n 432. 
no ha tenido lugar en el monte, sino en Matute. Sin embargo, repercute inmediatamente y de manera evidente para todos en las relaciones que se establecen en torno a la comunidad de pastos. Relacionar una cosa y otra no es un juego de manos de los monjes, interesados en que Colia siga poniendo cabañas. Pueden alegarse pruebas de otros lugares que confirman la percepción de una relación estrecha entre montes y homicidios. Hacia 1082, los de Albelda recogen la memoria de una serie de muertes, asesinatos y hallazgos de cadáveres producidos desde tiempo de García de Nájera en su territorio -en el monte, en el collado, en la peña, en el Ilano...- para demostrar que están exentos de pagar homicidio $^{90}$. En dos ocasiones, por un ladrón accidentalmente despeñado y un muerto causado por un barón con sus hombres, los de Albelda dicen literalmente que no pagaron homicidio pro termino, expresión que usan también con valor general al final del texto. Incluso en el celebérrimo texto de Berbea, Barrio y San Zadornil, de cronología incierta, edición troceada ${ }^{91}$ y crítica textual pendiente, puede observarse, de un lado que la exención de homicidio tiene un ámbito territorial supralocal -de plano de Erzezi ad sursum-, y de otro, que a la exención le sigue inmediatamente la afirmación, que en principio nadie había puesto en cuestión, de los usos de Barrio y Berbea en materia de montazgo. Con un método de análisis diferente, E. Peña y J.A. García de Cortázar han estudiado todas las referencias a exacciones y exenciones contenidas en la documentación riojana entre 1017 y 1076: no sólo el homicidio y las licencias de pastos constituyen los grupos más numerosos, sino que de las 22 exenciones de homicidio documentadas, diez están vinculadas a los pastos ${ }^{92}$.

\section{Conclusión}

El estudio del complejo territorial de Tobía -obispado, monasterios, comunidad- permite establecer algunos jalones de una trayectoria que arroja luz sobre el funcionamiento de este tipo de territorios en períodos de transición. Si la explicación del efímero obispado tobiense sólo resulta verosímil, en nuestra opinión, a partir de la existencia bajo el emirato de comunidades cristianas bien vertebradas y bien arraigadas en lo que será incorporado al reino de Pamplona en 923, los rasgos del monasterio de San Cristóbal en tiempo de Sancho el Mayor desvelan una implantación social y espacial vieja de algunas generaciones. Al final de esta línea hallamos comunidades que han estado al margen de la actividad de los grupos dirigentes de la España cristiana hasta su conquista y

90. UBIETO, CAlbelda, $\mathrm{n}^{\circ} 60$.

91. UBIETO, SMC I, no 67 y 145; LEDESMA, SMC II, n 107.

92. GARCÍA DE CORTÁZAR, J.Á., "La consolidación del dominio mental y físico del espacio riojano dentro del reino de Navarra entre 1016 y 1076" , en: Logroño (cit. n. 10) p. 93-129: p. 115-117. 
que tampoco parecen haber sufrido mayores impulsos transformadores por influencia de un poder islámico cuya penetración en la zona es débil. Nos remiten pues a dinámicas sociales y territoriales de un pasado difícil de precisar más allá de hablar de tardoantiguo o postvisigodo. La potencia de santuarios y reliquias como factor de cohesión es difícilmente discutible en un espacio donde las tumbas de San Prudencio, Santa Coloma y San Millán pueden visitarse prácticamente en una sola jornada a pie. Tampoco lo es su imbricación con una organización territorial en la que están orgánicamente unidos un espacio de hábitat y cultivos en que ambos muestran aún vestigios de movilidad, y un espacio de monte en el que hemos puesto preferentemente nuestra atención.

En efecto, el monte es un ámbito privilegiado para la vertebración política. En primer lugar, por su propio funcionamiento. Es un espacio exterior a la aldea sobre el que las comunidades ejercen un control mutuo constante, espoleado por el valor de sus recursos económicos y cuya materialización se renueva año a año con los desplazamientos de temporada del ganado ${ }^{93}$. Es decir, se trata de un territorio sobre el que se ejerce un juego de tensiones, de litigios y acuerdos, y sobre todo de mecanismos de contrapeso que preservan su carácter de espacio ajeno a la apropiación individual y sometido al control de todos. Lo cual es la definición misma del espacio público. Pero obsérvese bien que puede subsistir sin que exista necesariamente un poder superior al de la comunidad de pastos. Puede pues funcionar como elemento cohesionador de un territorio y de un conjunto de comunidades en períodos de transición.

En segundo lugar, por el filtro socioeconómico y jurídico que genera. Puesto que está vinculado a la vecindad, sólo los posesores de solares tienen derechos de explotación, lo que excluye a la población servil o dependiente -rasgo por otro lado común al conjunto de Occidente. Sobre esta igualdad jurídica, se da sin duda un aprovechamiento desigual de los recursos silvopastoriles. Recordemos las disensiones que se producen en la comunidad de Pazuengos en relación a la estrategia a seguir frente a San Millán. Muy probablemente es la franja superior de las comunidades la que llega a poseer grandes rebaños y por ende a controlar la comunidad de pastos ${ }^{94}$. Es decir, el grupo de familias que funcionan como engranaje entre el poder político y el medio local.

En 923 esta zona pasa a manos del reino de Pamplona. Una vez más podemos invocar el paralelismo con los símbolos de lo sagrado. Tan verosímil es el

93. En general, creemos que la flexibilidad del paisaje agrario al que hemos aludido más arriba, que implica la posibilidad de intensificar su explotación sin cambios en las formas de apropiación del territorio, y su vinculación orgánica con el espacio de montes, matiza la comprensión del crecimiento agrario en términos de una cierta competencia entre usos agrícolas y ganaderos: cf. MARTíN VISO, I., Poblamiento y estructuras sociales en el norte de la Península Ibérica. Siglos VI-XIII, Salamanca, 2000, p. 166 sq.

94. Cf. VIADER, “Maisons" (cit. n. 80). 
papel de reliquias como las de Santa Coloma, San Prudencio o San Millán en la cohesión interna de la comunidad bajo un poder no cristiano, como difícilmente discutible a un rey o a un conde su condición de protector de la Iglesia, sea enriqueciendo San Millán, sea asumiendo el patronato sobre Santa Coloma, sea vinculando San Prudencio a San Martín de Albelda. Tampoco es discutible su atributo como garante de la paz entre comunidades, lo que hace del monte el espacio en el que se solapan las dinámicas comunitarias supralocales y el poder real, con el añadido de que resulta especialmente eficaz, porque se vincula con una de las claves de arco de la apropiación del territorio. El homicidio pro termino viene así a formalizar y al tiempo a hacer visible uno de los modos en que se ha concretado esta articulación con el poder político.

Nótese en fin que de la caracterización del espacio de monte que hemos resumido en el párrafo anterior a la noción de fisco sólo va un tenue paso conceptual: se podría decir que sólo falta la presencia y la voluntad política de un rey. En este sentido, quizá estas páginas sobre Tobía y sus alrededores contribuyan a salvar la distancia que se deja ver entre quienes, estudiando la arquitectura política y territorial en tiempos de transición, han puesto el énfasis en las realidades comunitarias frente a quienes han privilegiado la materialización del fisco como instrumento del poder ${ }^{95}$.

95. Pensamos en particular en el sur del condado de Castilla, entre el Arlanza y el Duero. J. Escalona es posiblemente quien más énfasis ha puesto en el papel organizador de los que él llama territorios menores, en su estudio minucioso y en su comprensión a partir de las realidades locales. Subrayaremos aquí que también este autor atribuye un papel considerable a montes y dehesas en la articulación de estas comunidades con los poderes exteriores, y en particular con el condal (ESCALONA, J., Sociedad y territorio en la Alta Edad Media castellana. La formación del alfoz de Lara, Oxford, 2002, p. 87 sq.). Por su parte, E. Pastor ha enfocado en su análisis el modo en que la noción de fisco se materializa en el dominio condal sobre los espacios silvopastoriles (PASTOR, E., Castilla en el tránsito de la Antigüedad al feudalismo. Poblamiento, poder político y estructura social. Del Arlanza al Duero (siglos VII-XI), Valladolid, 1996, p. 159 sq.). 
OBISPOS EFÍMEROS, COMUNIDADES Y HOMICIDIO EN LA RIOJA ALTA EN LOS SIGLOS X Y XI
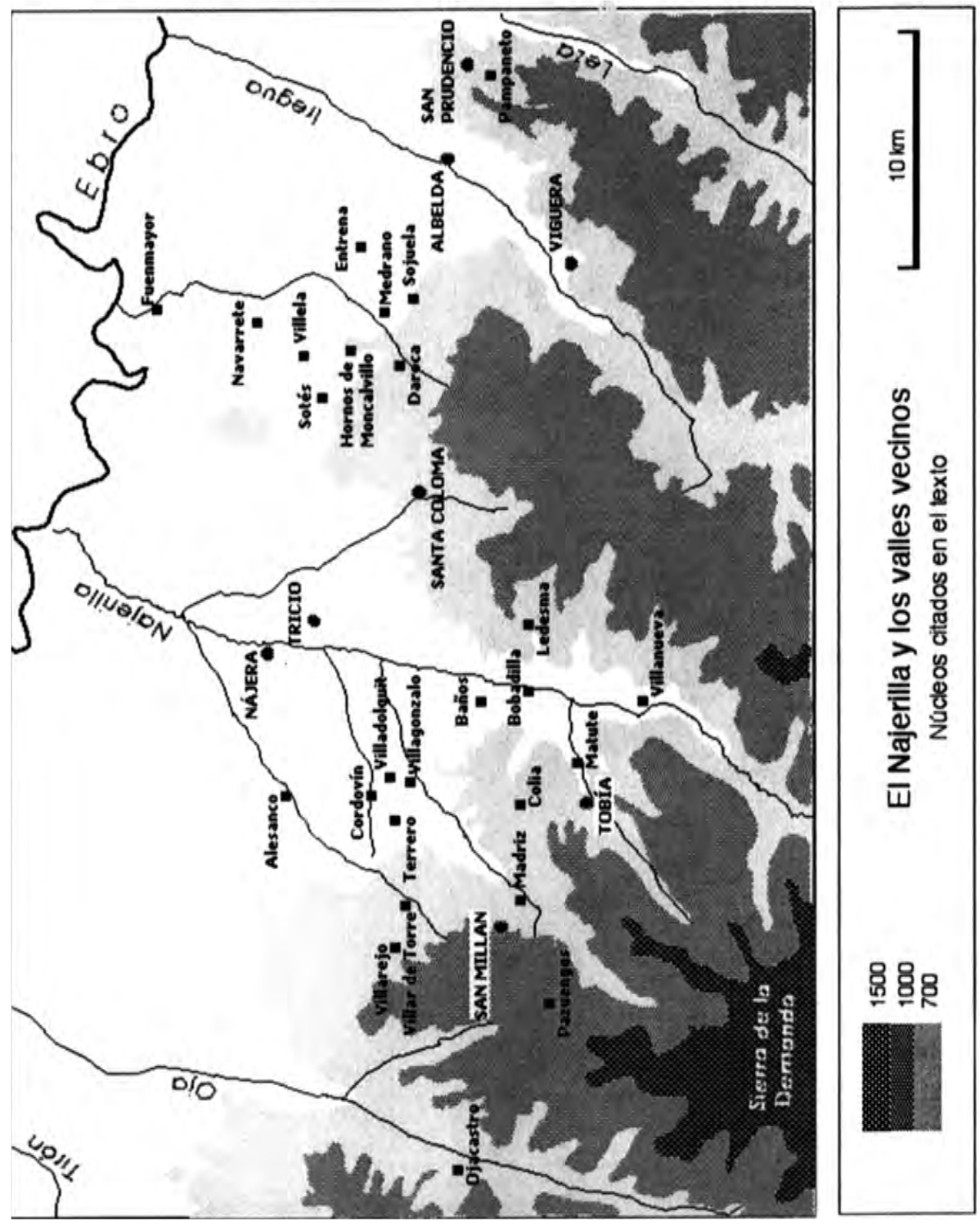